\title{
Differential Accessibility of a Hydrogenation Catalyst to Acyl Lipids in Thylakoid Membranes from Atrazine-Resistant and -Susceptible Solanum nigrum Biotypes
}

\author{
Jean-Philippe Mayor ${ }^{1}$, Zsolt Török ${ }^{2}$, Paul-André Siegenthaler ${ }^{1,3}$ \\ and Lászlo Vigh ${ }^{2}$ \\ 1 Laboratoire de Physiologie végétale, Université de Neuchâtel, Ch. de Chantemerle 20, \\ CH-2000 Neuchâtel, Switzerland \\ ${ }^{2}$ Institute of Biochemistry, Biological Research Centre, Hungarian Academy of Sciences, \\ P.O. Box 521, H-6701 Szeged, Hungary
}

\begin{abstract}
The novel water-soluble palladium complex was used as a probe to test the acyl lipid molecular organization of liposomes and thylakoids originating from triazine-resistant (R) and -susceptible (S) lines of Solanum nigrum. In the present study we report on differing susceptibility of some defined lipid classes towards hydrogenation which shows alterations in the accessibility of the catalyst to lipid substrates within R-compared to S-membranes. Further evidence to support differences in membrane architecture of S- and R-type thylakoids was provided by the lifetime distribution analysis of diphenylhexatriene. The remarkable relative increase of pool size and width of a medium lifetime decay component $(4 \mathrm{~ns})$ in S-samples indicates a more diverse molecular organization of this membrane. For an apparently identical lipid class and fatty acid composition and fluidity of thylakoids, the greater environmental heterogeneity of the fluorophore in $\mathrm{S}$-membranes may be attributed to stronger protein-lipid interactions. The possible existence of some intramembraneous domains of acyl lipids, some of which being relatively protected against the catalyst and present only in the atrazine-susceptible biotype, is discussed.
\end{abstract}

Key words: Acyl lipids - Fluorescence lifetime - Hydrogenation catalyst - Solanum nigrum Thylakoid membrane heterogeneity - Triazine-type herbicides.

The primary genetic and biochemical basis of triazine resistance of certain plant biotypes is rather well known. It has been shown that triazines compete with plastoquinone for a common binding site located on the D1 polypeptide of the PS II reaction center and that the differential response of resistant and susceptible biotypes to triazine treatment results from an altered affinity of the target protein towards the herbicide (Trebst 1987).

Besides those well-established facts, various data point out the importance of the lipid phase surrounding the herbicide binding polypeptide. For instance, early results by St.

Abbreviations: DGDG, digalactosyldiacylglycerol; DPH, diphenylhexatriene; $\mathrm{H}_{\mathrm{II}}$, hexagonal phase; LHCII, light harvesting complex proteins; MGDG, monogalactosyldiacylglycerol; PG, phosphatidylglycerol; PS II, photosystem II, R, resistant; S, susceptible, SQDG, sulfoquinovosyldiacylglycerol.

${ }^{3}$ To whom correspondence should be addressed.
John (1982) suggested that interaction between triazine herbicides and acyl lipids in chloroplast membranes may influence sensitivity of weed biotypes to the triazine herbicides. More recently, we have found that binding and inhibitory properties of triazine-herbicides undergo substantial changes upon specific lipid depletion by lipase treatments of thylakoids (Siegenthaler and Mayor 1992).

The existence of compositional and functional differences between thylakoid membranes originating from $\mathbf{R}$ and S-biotypes of $S$. nigrum further supports the involvement of acyl lipids in the mode of action of triazine-type herbicides. Among these differences are the variations found in the head group and fatty acid composition of membrane lipids (St. John 1982, Chapman et al. 1985). For instance, a higher content of 3 trans-hexadecenoic acid esterified in phosphatidylglycerol, which is thought to participate in the formation of the oligomeric structure of LHCII, was observed in the thylakoids of resistant bio- 
types, in parallel with a slightly increased unsaturation level of monogalactosyldiacylglycerol (St. John 1982, Lemoine et al. 1986). The molar ratio of lipid to protein, a factor determining the motional and thermotropic characteristics of biomembranes as well as the size of protein-immobilized lipid fraction, varied also when the two types were compared (Rawyler et al. 1987). Moreover, differences have been observed with respect to the amount of non-bilayer forming lipids in the two leaflets of thylakoids isolated from $\mathbf{R}$ - and S-type black nightshade species (Rawyler et al. 1987). Although the presence of widespread $\mathrm{H}_{1 \mathrm{II}}$ phases is rather unlikely under physiological conditions, the molecular properties which favour the formation of these phases may well be of structural and functional significance (Quinn et al. 1989).

A new approach is introduced here to probe for possible differences existing between the molecular organization of thylakoids originating from triazine- $R$ and $-S$ lines of Solanum nigrum. The method of homogeneous catalytical hydrogenation, exploiting the novel water-soluble palladium complex, has been successfully applied to remove acyl cis-double bonds from various intact biomembranes including chloroplast thylakoids (Vigh et al. 1985a, b, 1989, Quinn et al. 1989). In the present study we report on differing susceptibility of some defined lipid classes towards hydrogenation which suggests differences in the accessibility of the catalyst to acyl lipid substrates within R-and S-membranes. Furthermore, steady-state fluorescence polarization as well as the fluorescence decay of DPH has also been used to study the overall fluidity and microheterogeneity of both membrane types. The distributional analysis based on the maximum entropy method revealed significant differences between $\mathrm{S}$ - and $\mathbf{R}$-type thylakoids. The relatively increased environmental heterogeneity of the fluorophore in $S$-samples is in good accordance with reduced rate of catalytic hydrogenation.

\section{Materials and Methods}

Chemicals - The catalyst, palladium di (sodium alizarine monosulphonate), $\mathrm{PD}(\mathrm{QS})_{2}$, was purchased from Molecular Probes, Eugene, OR - DPH, 1,6-diphenyl-1,3,5-hexatriene was provided by Sigma. All other chemicals were of commercial laboratory grade and used without further purification.

Thylakoid preparation and hydrogenation procedure -Thylakoids were isolated from greenhouse-grown, freshly harvested leaves from black nightshade, Solanum nigrum L., according to Siegenthaler and Mayor (1992). After the final washing step $(1,500 \times g$ for $2 \mathrm{~min})$, thylakoids were resuspended in sorbitol $400 \mathrm{mM}$, MOPS-KOH $50 \mathrm{mM}, \mathrm{pH}$ 7.5, $\mathrm{MgCl}_{2} 2 \mathrm{mM}$, designated as "hydrogenation medium", and the chlorophyll concentration was adjusted to $0.06 \mathrm{mg}$

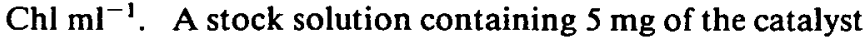

per $\mathrm{ml}$ of degassed hydrogenation medium was prepared simultaneously. Thereafter, the catalyst solution was preactivated before use by converting it to its hybride B-form (Pak et al. 1990), which permits hydrogenation without any induction period. Aliquots $(30 \mathrm{ml})$ of thylakoid suspensions were placed in $100 \mathrm{ml}$ custom designed reaction vessels, and transferred to a water bath at $+2{ }^{\circ} \mathrm{C}$. The reaction vessels were connected to a manifold and the gas phase evacuated and replaced by hydrogen, maintained at a pressure of 1 atmosphere. Aliquots of the preactivated B-form catalyst solution were then injected into the thylakoid suspension through a rubber septum to initiate the reaction. The final concentration of the Pd-complex was $0.1 \mathrm{mg} \mathrm{ml}^{-1}$. During the hydrogenation procedure (lasting a maximum of $5 \mathrm{~min}$ ), reaction vessels were rotated at $45 \mathrm{rev} \mathrm{min}^{-1}$. At the end of the desired incubations aliquots were removed from the vessel and the reaction was stopped by bubbling the suspension with air for $20 \mathrm{~s}$. After washing once with the hydrogenation medium to remove catalyst, thylakoids were harvested for lipid extraction (Vigh et al. 1989). Depigmented total thylakoid lipids were dispersed in the hydrogenation medium as described by Vigh et al. (1988) and subjected to hydrogenation according to the above procedure. Lipid analysis was carried out as reported by Siegenthaler et al. (1987) for phospholipids and by Rawyler and Siegenthaler (1985) for galactolipids. Polar lipids separated on pre-coated silica gel plates and their fatty acid methyl esters were prepared according to Vigh et al. (1988). Fatty acid methyl esters were analyzed isothermally $\left(180^{\circ} \mathrm{C}\right)$ on a Jeol JGK-20K gas chromatograph equipped with an SP-2330 capillary column (Vigh et al. 1988).

Estimation of membrane fluidity by measuring steadystate polarization of DPH fluorescence-Membrane fluidity was assessed by measurement of the motional freedom of the lipophylic probe, DPH according to Barber et al. (1984). DPH was added from a $3 \mathrm{mM}$ stock solution in tetrahydrofuran directly to a suspension of thylakoid membranes $\left(50 \mu \mathrm{g} \mathrm{Chl} \mathrm{ml}^{-1}\right)$ to a final concentration of $5 \mu \mathrm{M}$. Labelled suspensions were then incubated for $40 \mathrm{~min}$ in darkness at room temperature and washed by centrifugation. The pellet, resuspended in $400 \mathrm{mM}$ sorbitol, $0.2 \mathrm{mM}$ $\mathrm{MgCl}_{2}, 5 \mathrm{mM}$ MOPS ( $\mathrm{pH} 7.5$ ), was then used for measurements. Steady-state polarization was measured by applying a Hitachi MPF-2A spectrofluorometer equipped with polarization filters. Temperature in the cuvettes was maintained with a cuvette holder thermostated by a computercontrolled Peltier device. The measured fluorescence intensities were corrected for background fluorescence and light scattering from the untreated sample. The degree of polarization (p) was calculated according to Barber et al. (1984). All the above measurements were performed at least in triplicate.

Membrane microheterogeneity studies using DPH fluo- 
rescence lifetime distribution-DPH-labelling of thylakoid suspension was performed as described for steady-state measurements. Fluorescence lifetime measurements were carried out with a PTI LS-100 nanosecond fluoresence spectrophotometer (Photon Technology International Inc., London, Canada). Excitation light at $358 \mathrm{~nm}$ was provided by a thyratron-gated $\mathrm{N}_{2}$-filled flash lamp. Fluorescence decays were measured at $460 \mathrm{~nm}$. The sample temperature $\left(2^{\circ} \mathrm{C}\right)$ was controlled by using an external bath circulator. The data analysis was achieved by applying the software of Photon Technology International based on the maximum entropy method (MEM) for the lifetime distribution analysis (Siemiarczuk et al. 1990).

\section{Results and Discussion}

Thylakoids isolated from R- and S-biotype were submitted to catalytic hydrogenation and the kinetics of double bond reduction was monitored by fatty acid analysis of total lipids. Fig. 1A shows that under the above-selected conditions, the double bond content decreased appreciably, reaching its minimum after $5 \mathrm{~min}$ of exposure to B-form catalyst. Extensive differences can be revealed between the saturation curves of R- and S-biotypes, particularly in briefly hydrogenated samples. Although both plots were biphasic, i.e., an apparent lag phase was followed by the main reaction, significant hydrogenation began only about $50 \mathrm{~s}$ later in S-compared to R-thylakoids. This feature resulted in a well-reproducible difference in the extent of saturation between the two samples $(30-40 \%$ at 60 $120 \mathrm{~s}$ range), which was maintained, though to a lesser extent, at the end of the reactions. When liposomes were formed from thylakoid polar lipids and submitted to a similar hydrogenation procedure, R- and S-samples displayed identical saturation curves (Fig. 1B). This finding indicates that, following the abolishment of the original membrane assembly, the total polar lipids became equally accessible to the catalyst.

To obtain more detailed insight into the relative "protection" of lipids towards the catalyst within native thylakoids from $\mathrm{R}$ - and S-biotypes, the hydrogenation of individual lipid classes was studied at various time intervals (Fig. 2). The patterns of hydrogenation of glycolipids (MGDG, DGDG, SQDG) again revealed a marked difference between R- and S-membranes. Galactolipids and SQDG were more readily hydrogenated in the thylakoids of atrazine-resistant biotype (Figs. 2A-C). Surprisingly, the rates of catalytic hydrogenation of $P G$ were similar in both types of membranes (Fig. 2D). This observation indicates that those factors rendering some glycolipid pools relatively less accessible to the catalyst in S-type membranes are inoperative regarding PG.

Current evidence derived from a wide range of applications of Pd-catalyst in model- and biomembranes has sug- gested that differences in apparent accessibility of the amphipathic hydrogenation catalyst to lipid substrates could be explained by at least three main reasons (Joò et al. 1991): (i) an increase of the bulk membrane microviscosity (Quinn et al. 1989, Vigh et al. 1983), (ii) the existence of lipid microdomains due to lateral heterogeneity in membranes (Katagiri et al. 1981, Schlame et al. 1990), (iii) a phase separation of non-bilayer lipids forming $\mathrm{H}_{\mathrm{II}}$ phase (Vigh et al. 1987).

In contrast to the original concept of fluid mosaic model, theoretical considerations and experimental evidence suggest a non-random distribution of both membrane lipids and proteins. Although the evidence for biological membrane structural heterogeneity is indirect at present (Fiorini et al. 1987), lipid microdomains may be thermodynamically stable within biomembranes. Lateral phase separation or immiscibility of lipids as well as the presence of proteins (Williams et al. 1990) may have a primary role in the stabilization of membrane microhetero-
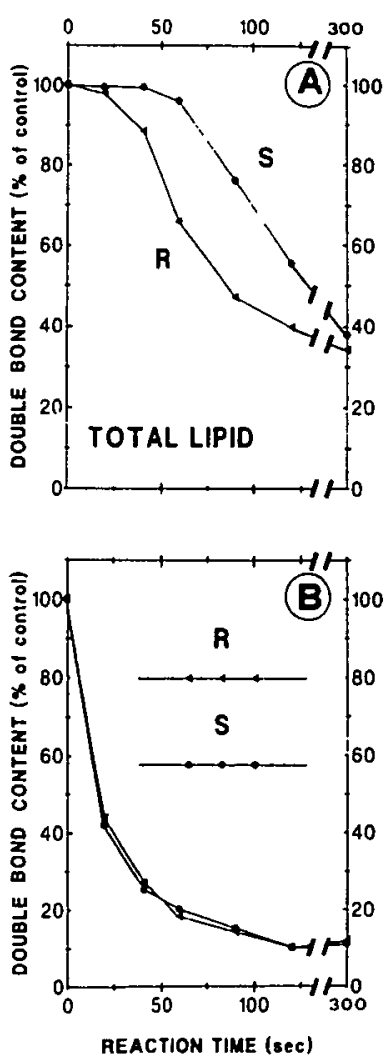

Fig. 1 Effect of homogenous catalytic hydrogenation on the decrease of total fatty acid double bonds in thylakoids (A) or in liposomes constituted of thylakoid total polar lipids (B) originating from triazine-susceptible (S) and resistant (R) Solanum nigrum biotypes. $100 \%$ values corresponded to a double bond index of 2.53 for both biotypes. For further details, see Materials and Methods. 
geneity. Based on the great sensitivity of DPH lifetime to monitor the physical state of the membrane, this probe can be applied to reveal the relative amount of membrane microdomains. DPH molecules exist in a variety of different positions along the normal membrane and because they undergo rapid rotational and translational motions, they can experience a range of environments, each of which is characterized by different lifetime values. Although the exact basis for the environmental heterogeneity caused by membrane proteins has to be ascertained, evidence for a distinct heterogeneity in the environment of DPH in the protein-lipid interfacial region has been reported recently (Williams et al. 1990). Since we postulated that protein-induced immobilization of some lipids could result in a delay in their catalytic hydrogenation, we investigated membrane organization in thylakoids derived from R-and S- Solanum nigrum biotypes by analysing the heterogeneity of DPH decay.

To assess the contribution of the above listed possibilities to the differential accessibility of Pd-catalyst to gly-
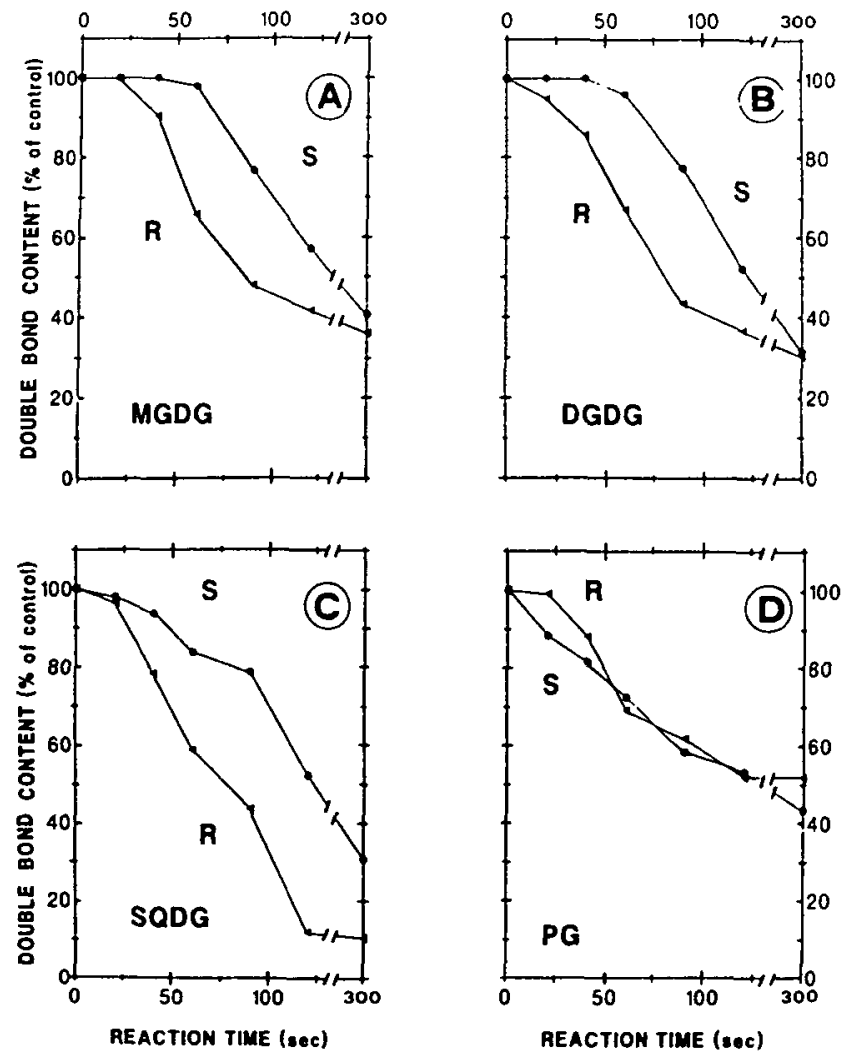

Fig. 2 Change in fatty acid unsaturation of individual polar lipid classes following the hydrogenation of S- and R-type thylakoids. $\quad 100 \%$ corresponded in the $\mathrm{S}$ - and R-biotypes to a double bond index of 2.72 and 2.84, respectively, for MGDG (A); 2.51 and 2.50 for DGDG (B); 1.40 and 1.28 for SQDG (C); 1.20 and 1.15 for PG (D). colipids, we first determined the overall fluidity of membranes. Fig. 3 shows that upon screening over a wide temperature range $\left(5-50^{\circ} \mathrm{C}\right)$, no significant differences of the polarization values were found between $\mathbf{R}$ - and S-biotype thylakoids. Moreover, the same degree of fatty acid unsaturation was found prior to hydrogenation (double bond index $2.53 \pm 0.04$ ) in total thylakoid lipids originating either from triazine-R or -S-biotype. Although minor differences in the amount of lipid classes could be observed between R-and S-type samples with respect to SQDG (data not shown), these may be considered negligeable. Thus, the observed shielding of glycolipids towards the Pd-complex cannot be related to an altered bulk membrane fluidity of S-type thylakoids.

To investigate at a lower molecular level the organization of R- and S-type thylakoids, DPH decays were analysed using the continuous lifetime distribution approach. To obtain a good fit, lifetime distribution of both membranes could be resolved into 3 components: (1) a short, (2) a medium and (3) a relatively long one (Fig. 4). The exact data of the center, width at half-maximum and fractional intensity of each components are presented in Table 1. In the case of long life-time components $\left(\mathrm{C}_{3}\right)$, the center of the distribution (11.0 ns) as well as the W values $(0.7 \mathrm{~ns})$ are identical for both biotypes. In contrast, both the medium $\left(C_{2}\right)$ and short $\left(C_{1}\right)$ components displayed significantly longer $\mathrm{C}$ and $\mathrm{W}$ values in S-type samples. Whereas the fraction size of short components was practically the same, $f_{2}$ and $f_{3}$ were remarkably different in the two biotypes. The fractional intensity of long lifetime component in Stype samples was about $25 \%$ lower than the corresponding value in R-type. In contrast, a comparison of the size of medium lifetime components revealed the opposite tenden-

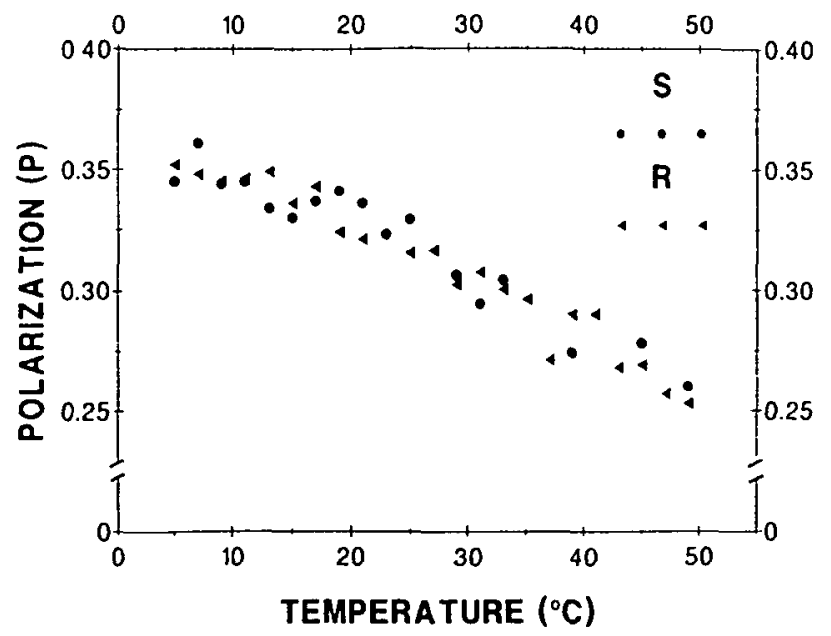

Fig. 3 Steady-state polarization values (P) of DPH fluorescence at various temperatures in thylakoid membranes isolated from atrazine-susceptible and atrazine-resistant $S$. nigrum biotypes. 
Table 1 Distribution analysis of DPH fluorescence decay in freshly prepared thylakoid membranes from R- and SSolanum nigrum biotypes

\begin{tabular}{|c|c|c|c|c|c|c|c|c|c|c|}
\hline & \multicolumn{3}{|c|}{$C_{1}$ component } & \multicolumn{3}{|c|}{$C_{2}$ component } & \multicolumn{4}{|c|}{$\mathrm{C}_{3}$ component } \\
\hline & $\mathrm{C}_{1}$ & $\mathrm{~W}_{1}$ & $f_{1}$ & $\mathrm{C}_{2}$ & $\mathrm{~W}_{2}$ & $f_{2}$ & $\mathrm{C}_{3}$ & $\mathrm{~W}_{3}$ & $f_{3}$ & $\chi^{2}$ \\
\hline S-type & 0.23 & 0.33 & 0.08 & 4.0 & 3.1 & 0.53 & 11.0 & 0.7 & 0.39 & 1.22 \\
\hline R-type & 0.21 & 0.29 & 0.07 & 2.9 & 1.8 & 0.41 & 11.0 & 0.7 & 0.52 & 1.24 \\
\hline
\end{tabular}

(C, center of the lifetime distribution (in ns) of the short $\left(C_{1}\right)$, medium $\left(C_{2}\right)$ and long $\left(C_{3}\right)$ components; $W$, full width at half maximum in $\mathrm{ns}$; f, fractional intensity; $\chi^{2}$, reduced chi-square).

cy: $f_{2}$ value in $R$-type thylakoids was about three-quarters of the corresponding $f_{2}$ value in S-type sample. It is known that the width of the recovered distribution gives an indication of the degree of environmental heterogeneity of the fluorophore (Williams et al. 1990). On the basis of the strikingly different distribution width of $\mathrm{C}_{2}$ components, we can conclude that the S-type membrane is more heterogeneous than the R-one. Therefore, this finding may explain the relatively delayed rate of hydrogenation of Smembrane lipids.

A last important question is the exact location and reason of the environmentally diverse regions sampled by DPH. The majority of previous distributional studies of DPH fluorescence decay was based on double-exponential and bimodal Lorentzian analyses. By applying the bimodal approach in our case the fit showed significantly larger $\chi^{2}$ than by resolving the decay for three continuous distributional components (data not shown). . This finding suggests that the two-component model may not be ade-

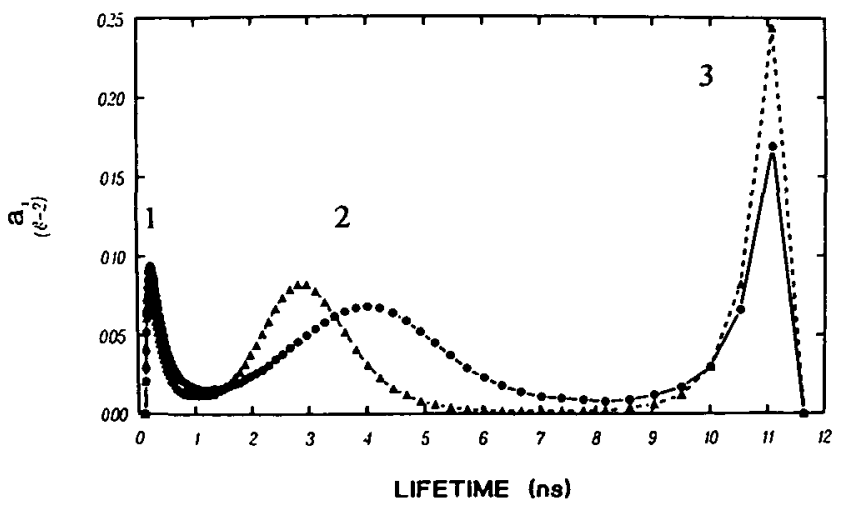

Fig. 4 DPH lifetime distribution in thylakoids derived from atrazine-resistant (- $\left.\mathbf{A}^{-}\right)$and -susceptible (-) biotypes. The $a_{i}$ is a preexponential factor representing the fractional contribution to the time-resolved decay of the component with a lifetime, according to Siemiarczuk et al. 1990. The three main lifetime components are indicated by numbering (for details, see also the text and Table 1). quate to describe the DPH decay for our samples.

The origin of the short $\left(\mathrm{C}_{1}\right)$ components, which was apparently identical in our two samples, has been extensively discussed. It has been assigned to a fraction of photolytic products to probe molecules located in a very polar environment at the membrane surface or to defects between membrane domains (Zolese et al. 1990).

We suppose that the long lifetime component may derive from lipids located in protein-poor regions. This can be inferred both from the relatively high $\mathrm{C}_{3}$ and narrow width values (Williams et al. 1990).

The decrease in DPH lifetime in $\mathrm{C}_{2}$ region could be an indication of the energy transfer between the fluorophore and the intrinsic membrane proteins (Williams et al. 1990). Based on this fact and on the strikingly different distribution width, we postulate, that these remaining medium components represent the boundary layer of lipids interacting with proteins. It was predicted, that proteinimmobilization of lipids reduces their accessibility to Pd-catalyst in native membranes (Schlame et al. 1990). Therefore, this approach provides a ready explanation for the differential kinetics of lipid hydrogenation obtained in the present study. The relatively increased fraction size of protein-immobilized lipid pool in S-type thylakoids would also be in accordance with the significantly higher proteinto-lipid ratio (see later). It should be added, that due to the almost identical lipid and fatty acid composition of the two kinds of membranes, the increase in distributional width of $\mathrm{C}_{2}$ in S-sample cannot be attributed to the compositional diversity of lipids.

The lipid-protein association question-Although there are various proposals for the molecular topography of the thylakoid membrane, considerable uncertainty still exists about the size of tightly-bound lipid shells around the proteins. Lipid-protein interactions in thylakoid and subthylakoid fractions have been studied by using spin-labelled analogues of membrane lipid components. For instance, spin-labelled MGDG exhibited a less preferential interaction over $P G$, suggesting that part of the role of MGDG is structural in thylakoid membranes ( $\mathrm{Li}$ et al. 1989). According to another investigation, the fraction of 
motionally restricted lipids was found to be qualitatively in line with the protein-to-lipid ratio values of different thylakoid membranes ( $\mathrm{Li}$ et al. 1990). Previous studies with $S$. nigrum indicated that the protein/lipid ratio was significantly higher in the herbicide-sensitive biotype, e.g., 2.70 vs $2.33 \mathrm{mg}$ protein $\mu \mathrm{mol}^{-1}$ acyl lipid in the resistant biotype (Rawyler et al. 1987). Provided that the amount of protein-immobilized glycolipids is also higher in the Stype thylakoid membranes, their relatively restricted access by the catalyst is conceivable. To assess the validity of this hypothesis, investigations are presently underway to quantify the proportion of protein-immobilized glycolipids in thylakoids of $S$. nigrum biotypes by using spin-labelled MGDG (12-MGDGSL). In support of our concept, a reduced access of Pd-catalyst to specific cardiolipin pools, tightly associated with integral mitochondrial proteins, has been observed recently (Schlame et al. 1990). Furthermore, the association of cardiolipin with membrane proteins becomes looser upon double bond reduction of the bulk lipids resulting in a greater accessibility of the applied catalyst to cardiolipins at a more advanced stage of hydrogenation. Similar mechanisms may govern acyl lipid hydrogenation in the present case.

The hexagonal phase question-The transmembrane distribution of MGDG and DGDG has been determined from both type of biotypes of S. nigrum (Rawyler et al. 1987). Whereas the molar ratios of the two main galactolipid classes (MGDG/DGDG) are practically identical in the inner leaflet (R: 1.32; $S: 1.24$ ) there is a significant difference in the outer monolayer of R-and S-type thylakoids (R: 7.00; S: 9.67). Due to this striking higher ratio in the outer leaflet of S-thylakoids, one can link this finding to a higher probability of acyl lipids to form non-lamellar structures (Giroud and Siegenthaler 1988), as being a potential factor restricting the accessibility of the catalyst (Vigh et al. 1987). However, at least three major objections can be raised against this assumption. Firstly, we found a lag phase and a reduced extent of catalytic hydrogenation reactions in all glycolipid classes in S-membranes (Fig. 2), although it is anticipated that DGDG, a bilayer forming lipid is essentially excluded from $\mathrm{H}_{\mathrm{II}}$ domains (Quinn et al. 1989). Secondly, lamellar to non-bilayer phase separation is presumed to occur by exposing membranes to temperatures higher than those of the physiological range (Quinn 1989, Vigh et al. 1989, Quinn et al. 1989, Horvath et al. 1986); in this investigation, hydrogenation reactions were performed at $+2^{\circ} \mathrm{C}$. Finally, increased heat-susceptibility of triazine-resistant lines of $S$. nigrum has been documented (Ducruet and Ort 1988, Havaux 1989). Since the appearance of non-bilayer structures can be directly linked to the reduced thermal stability under heat stress (Quinn 1989 , Vigh et al. 1989) it is hard to believe that hydrogenation of S-type thylakoids could be limited by nonbilayer lipid phase. Another interesting point would be to clarify the mechanism of elevated heat-tolerance of S-biotype. Indeed, increased heat tolerance of photosynthetic activity was directly linked to the double bond content of fatty acyl chains as shown in pea thylakoids (Vigh et al. 1989, Quinn et al. 1989).

In summary, our results strongly suggest the existence of different intramembraneous compartmentations of acyl lipids within thylakoid membranes of atrazine-resistant and -susceptible weed biotypes. Further efforts are needed to understand the mechanism of their formation and to estimate their biochemical and physiological significance. The proper description of the difference between the thylakoid molecular organization of the two biotypes could bring a new perspective in evaluating the competitive ability of weed populations differing in triazine-resistance as well as in the prospect of breeding triazine-resistant crop cultivars.

The authors are grateful to Mr. Dominique Monney and to Mrs. Zs. Gazó for their skillful technical assistance. This study was supported by the Swiss National Science Foundation (Grants Nos 3.346-0.86 and 31.26386.89 to P.A.S.) and by the Hungarian National Scientific Research Foundation (OTKA No. 897). This work is part of a doctoral program which is carried out by J.P.M. in the Laboratoire de Physiologie végétale, Université de Neuchâtel).

\section{References}

Barber, J., Ford, R. C., Mitchell, R. A. C. and Millner, P. A. (1984) Chloroplast thylakoid membrane fluidity and its sensitivity to temperature. Planta 161: 375-380.

Chapman, D. J., De-Felice, J. and Barber, J. (1985) Characteristics of chloroplast thylakoid lipid composition associated with resistance to triazine herbicides. Planta 166: 280-285.

Ducruet, J.M. and Ort, D. (1988) Enhanced susceptibility of photosynthesis to high leaf temperature in triazine-resistant Solanum nigrum L. Evidence for photosystem II $\mathrm{D}_{1}$ protein site of action. Plant Sci. 56: 39-48.

Fiorini, R., Valentino, M., Wang, S., Glaser, M. and Gratton, E. (1987) Fluorescence lifetime distributions of 1,6-diphenyl1,3,5-hexatriene in phospholipid vesicles. Biochemistry 26 : 3864-3870.

Giroud, C. and Siegenthaler, P. A. (1988) Development of oat prothylakoids into thylakoids during greening does not change transmembrane galactolipids asymmetry but preserves the thylakoid bilayer. Plant Physiol. 88: 412-417.

Havaux, M. (1989) Comparison of atrazine-resistant and -susceptible biotypes of Senecio vulgaris L.: Effects of high and low temperatures on the in vivo photosynthetic electron transfer in intact leaves. J. Exp. Bot. 40: 849-854.

Horváth, I., Mansourian, A. R., Vigh, L., Thomas, P. G., Joó, F. and Quinn, P. J. (1986) Homogeneous catalytic hydrogenation of the polar lipids of pea chloroplasts in situ and the effects 
on lipid polymorphism. Chem. Phys. Lipids 39: 251-264.

Joó, F., Balogh, N., Horváth, L. I., Filep, G., Horváth, I. and Vigh, L. (1991) Complex hydrogenation/oxidation reactions of the water-soluble hydrogenation catalyst palladium di (sodium alizarin monosulfonate) and details of homogeneous hydrogenation of lipids in isolated biomembranes and living cells. Anal. Biochem. 194: 34-40.

Katagiri, C., Owen, J. S., Quinn, P. J. and Chapman, D. (1981) Hydrogenation of plasma lipoproteins by water-soluble catalyst; its use as a structural probe. Eur. J. Biochem. 118: 335338.

Lemoine, Y., Dubacq, J.-P., Zabulon, G. and Ducruet, J.-M. (1986) Organization of the photosynthetic apparatus from triazine-resistant and -susceptible biotypes of several plant species. Can. J. Bot. 64: 2999-3007.

Li, G., Knowles, P. F., Murphy, D. J. and Marsh, D. (1990) Lipid-protein interactions in thylakoid membranes of chillingresistant and chilling-sensitive plants studied by spin label electron spin resonance spectroscopy. J. Biol. Chem. 265: 1686716872.

Li, G., Knowles, P. F., Murphy, D. J., Nishida, I. and Marsh, D. (1989) Spin-label ESR studies of lipid-protein interaction in thylakoid membranes. Biochemistry 28: 7446-7452.

Pak, Y., Joó, F., Vigh, L., Katho, A. and Thompson, G. A., Jr. (1990) Action of a homogeneous hydrogenation catalyst on living Tetrahymena mimbres cells. Biochim. Biophys. Acta 1023: 230-238.

Quinn, P. J. (1989) Principles of membrane stability and phase behavior under extreme conditions. J. Bioenerg. Biomembr. 21: 3-19.

Quinn, P. J., Joó, F. and Vigh, L. (1989) The role of unsaturated lipids in membrane structure and stability. Prog. Biophys. Molec. Biol. 53: 71-103.

Rawyler, A. and Siegenthaler, P.-A. (1985) Transversal localization of monogalactosyldiacylglycerol and digalactosyldiacylglycerol in spinach thylakoid membranes. Biochim. Biophys. Acta 815: 287-298.

Rawyler, A., Unitt, M. D., Giroud, C., Davies, H., Mayor, J.-P., Harwood, J. L. and Siegenthaler, P.-A. (1987) The transmembrane distribution of galactolipids in chloroplast thylakoids is universal in a wide variety of temperate climate plant. Photosyn. Res. 11: 3-13.

Schlame, M., Horváth, L. I. and Vigh, L. (1990) Relationship between lipid saturation and lipid-protein interaction in liver mitochondria modified by catalytic hydrogenation with reference to cardiolipin molecular species. Biochem. J. 265: 79-85.

Siegenthaler, P.-A. and Mayor, J.-P. (1992) Changes in the binding and inhibitory properties of urea/triazine-type herbicides upon phospholipid and galactolipid depletion in the outer monolayer of thylakoid membranes. Different behaviour of atrazine-susceptible and -resistant biotypes of Solanum nigrum
L. Photosyn. Res. 31: 57-68.

Siegenthaler, P.-A., Smutny, J. and Rawyler, A. (1987) Involvement of distinct populations of phosphatidylglycerol and phosphatidylcholine molecules in photosynthetic electron-flow activities. Biochim. Biophys. Acta 891: 85-93.

Siemiarczuk, A., Wagner, B. D. and Ware, W. R. (1990) Comparison of the maximum entropy and exponential series methods for the recovery of distributions of lifetimes from fluorescence lifetime data. J. Phys. Chem. 94: 1661-1666.

St. John, J. B. (1982) Effects of herbicides on the lipid composition of plant membranes. In Biochemical Responses Induced by Herbicides. Edited by Moreland, D. E., St. John, J. B. and Hess, F. D. pp. 97-109. ACS Symposium series 181, American Chemical Society, Washington DC 1982.

Trebst, A. (1987) The three-dimensional structure of the herbicide binding niche on the reaction center polypeptides of photosystem II. Z. Naturforsch. 42c: 742-750.

Vigh, L., Gombos, Z., Horváth, I. and Joó, F. (1989) Saturation of membrane lipids by hydrogenation induces thermal stability in chloroplast inhibiting the heat-dependent stimulation of photosystem I-mediated electron transport. Biochim. Biophys. Acta 979: 361-364.

Vigh, L., Gombos, Z. and Jo6, F. (1985a) Selective modification of cytoplasmic membrane fluidity by catalytic hydrogenation provides evidence on its primary role in chilling susceptibility of the blue-green alga Anacystis nidulans. FEBS Lett. 191: 200204.

Vigh, L., Horváth, I., Joó, F. and Thompson, G. A., Jr. (1987) The hydrogenation of phospholipid-bound unsaturated fatty acids by homogeneous, water-soluble, palladium catalyst. Biochim. Biophys. Acta 921: 167-174.

Vigh, L., Horváth, I. and Thompson, G. A., Jr. (1988) Recovery of Dunaliella salina cells following hydrogenation of lipids in specific membranes by homogeneous palladium catalyst. Biochim. Biophys. Acta 937: 42-50.

Vigh, L., Joó, F., Droppa, M., Horváth, L. I. and Horváth, G. (1985b) Modulation of chloroplast membrane lipids by homogeneous catalytic hydrogenation. Eur. J. Biochem. 147: 477481 .

Vigh, L., Joó, F., van Hasselt, P. R. and Kuiper, P. J. C. (1983) Hydrogenation of model and biomembranes using a water-soluble ruthenium phosphine catalyst. J. Mol. Catal. 22: 15-19.

Williams, B. W., Scotto, A. W. and Stubbs, C. D. (1990) Effect of proteins on fluorophore lifetime heterogeneity in lipid bilayers. Biochemistry 29: 3248-3255.

Zolese, G., Gratton, E. and Curatola, G. (1990) Phosphatidic acid affects structural organization of phosphatidylcholine liposomes. A study of 1,6-diphenyl-1,3,5-hexatriene (DPH) and 1-(4-trimethylammonium-phenyl)-6-phenyl-1,3,5hexatriene (TMA-DPH) fluorescence decay using distributional analysis. Chem. Phys. Lipids 55: 29-39.

(Received October 15, 1991; Accepted January 13, 1992) 
